\title{
BIAS: A Toolbox for Benchmarking Structural Bias in the Continuous Domain
}

This paper was downloaded from TechRxiv (https://www.techrxiv.org).

\section{LICENSE}

CC BY 4.0

SUBMISSION DATE / POSTED DATE

09-09-2021 / 11-09-2021

\section{CITATION}

Vermetten, Diederick; van Stein, Bas; Caraffini, Fabio; Minku, Leandro; Kononova, Anna V. (2021): BIAS: A Toolbox for Benchmarking Structural Bias in the Continuous Domain. TechRxiv. Preprint. https://doi.org/10.36227/techrxiv.16594880.v1

$\mathrm{DOI}$

10.36227/techrxiv.16594880.v1 


\title{
BIAS: A Toolbox for Benchmarking Structural Bias in the Continuous Domain
}

\author{
Diederick Vermetten, Bas van Stein, Fabio Caraffini, Member, IEEE, Leandro L. Minku, Senior Member, IEEE \\ and Anna V. Kononova, IEEE Member
}

\begin{abstract}
Benchmarking heuristic algorithms is vital to understand under which conditions and on what kind of problems certain algorithms perform well. Most benchmarks are performance-based, to test algorithm performance under a wide set of conditions. There are also resource- and behaviour-based benchmarks to test the resource consumption and the behaviour of algorithms. In this article, we propose a novel behaviourbased benchmark toolbox: BIAS (Bias in Algorithms, Structural). This toolbox can detect structural bias per dimension and across dimension based on 39 statistical tests. Moreover, it predicts the type of structural bias using a Random Forest model. BIAS can be used to better understand and improve existing algorithms (removing bias) as well as to test novel algorithms for structural bias in an early phase of development. Experiments with a large set of generated structural bias scenarios show that BIAS was successful in identifying bias. In addition we also provide the results of BIAS on 432 existing state-of-the-art optimisation algorithms showing that different kinds of structural bias are present in these algorithms, mostly towards the centre of the objective space or showing discretization behaviour. The proposed toolbox is made available open-source and recommendations are provided for the sample size and hyper-parameters to be used when applying the toolbox on other algorithms.
\end{abstract}

\section{INTRODUCTION}

The modern world has an ever growing need for good heuristic optimisation algorithms due to the large amounts of data and increasingly difficult problems to be optimised. Since one overall best heuristic does not exist [1], we have to benchmark heuristics to understand which one is better under what conditions. Benchmarking can be performancebased, resources-based or behaviour-based. Most benchmarks for continuous optimisation are performance-based. An example is the well-known Black-Box Optimisation Benchmark (BBOB) [2]. Performance-based benchmarks aid in learning about the performance of one algorithm and the comparison between other algorithms in different situations. For example, one algorithm could perform very well on separable functions and another algorithm on uni-modal, high-conditioned functions. Resource-based benchmarks show the amount of resources (computation power / memory / energy) required under certain conditions. However, these types of benchmarks do not easily allow the analysis of the 'behaviour' of these algorithms under different circumstances. Behaviour is, for

Manuscript received September 9, 2021 (Corresponding author: Diederick Vermetten). Diederick Vermetten (d.1.vermetten@liacs.leidenuniv.nl), Bas van Stein and Anna V. Kononova are with the Leiden Institute of Advanced Computer Science, University Leiden, The Netherlands. Fabio Caraffini is with the School of Computer Science and Informatics, De Montfort University, Leicester, UK and Leandro L. Minku is with the School of Computer Science, University of Birmingham, UK. example, how a population of candidate solutions move in a swarm optimisation algorithm either dependent or independent of the function to optimise.

An example of a behaviour-based benchmark is RobustBench [3], an adversarial attack benchmark designed to test the robustness of image classification deep neural networks. Behaviour-based benchmarks can be used to learn additional information about the behaviour of an algorithm under different conditions. Here, we concentrate on a particular kind of behaviour-based performance estimate, Structural Bias (SB). $\mathrm{SB}$ is a form of bias inherent to the iterative heuristic optimiser in the objective space that also affects the performance of the optimisation algorithm.

Detecting whether, when and what type of SB occurs in a heuristic optimisation algorithm can provide guidance on what needs to be improved in these algorithms, besides helping to identify conditions under which such bias would not occur. In many cases SB can be avoided by slightly redesigning an algorithm component or by using different hyper-parameters. However, SB is hard to detect when not specifically looking for these kind of issues. Therefore, in this paper we propose a toolbox to automatically benchmark continuous optimisation algorithms to discover a portfolio of eleven different SB scenarios. We aim to answer the following research questions:

RQ1 How to determine whether a heuristic continuous optimisation algorithm suffers from SB?

RQ2 How to determine the type of SB suffered by a heuristic continuous optimisation algorithm?

RQ3 Which heuristic continuous optimisation algorithms suffer from what type of SB? Under what conditions?

To answer RQ1, we evaluate a large set of statistical tests based on 1500 repetitions of 194 different artificially generated parameterised distributions containing 11 different scenarios of structural bias. The proposed toolbox can be used to detect potential bias issues in newly developed algorithms as well as benchmark already existing optimisation algorithms. To answer RQ2, we propose a machine learning approach to identify which SB scenarios are most likely occurring in a given optimiser based on the resulting test statistics. The complete SB benchmark statistical test suite (BIAS), data generators for different SB scenarios and the machine learning model for identifying different SB scenarios are provided open-source [4]. To answer RQ3, we adopt the proposed toolbox to detect SB in 432 different continuous optimisation algorithms. We also discuss insights provided by the resulting benchmarking of these algorithms and potential future research 
directions.

The paper is structured as follows: in Section II, SB is introduced and explained in detail. The main components of our proposed BIAS toolbox are also briefly introduced. In Section III, all considered statistical tests for uniformity are introduced, including several newly proposed tests specifically for SB. In Section IV, the SB scenarios and experiments are explained in detail. In Section V, an analysis of the statistical tests is conducted, answering RQ1. In Section VI, the machine learning approach used to identify the type of SB is introduced and evaluated, answering RQ2. In Section VII, existing heuristic continuous optimisation algorithms are benchmarked, answering RQ3. Finally, we end with recommendations and conclusions in Section VIII.

\section{Structural Bias}

Put simply, algorithms are tools applied to particular problems. Just like a good hammer working well on many nails, we would like algorithms to ideally deliver good solutions for many problems. However, as postulated by the so-called No-Free Lunch Theorem, no best optimiser exists over all possible optimisation problems [1]. What is possible though is algorithms specialising on certain kind of problems. Identifying sets of such suitable problems for each algorithm is not a trivial problem [5] and many landscape features have been investigated to understand the success of some algorithms on some kinds of functions [6]. However, what cannot practically be a feature uniting such problems is the location of optima in the domain - having an algorithm that consistently finds an optima only located in the origin is of no use. Therefore, good algorithms should not be biased towards specific locations of the search space, e.g., towards solutions at the origin, centre, or in the borders of the search space. Extrapolating such reasoning, a good optimisation algorithm should be able to find the optima regardless where exactly they are located within the domain. Or, even stronger, a good algorithm should ideally locate solutions anywhere in its domain with equal 'effort'.

In case of iterative optimisation algorithms, points sampled during the initialisation 'move' within the domain defined by its boundaries under the influence of algorithm's operators and potentially bring improvement of the values of the objective function during the optimisation run, following some kind of 'survival-of-the-fittest' logic. In effect, such movement of the algorithm towards the optima gets steered by the differences in the values of the objective function in the sampled points or their derivates of some kind. Any feedback that is external to the objective function or domain knowledge might hinder such progression to the optima. Such external feedback stemming from the iterative nature of the algorithm is referred to here as structural bias.

Because of the high interdependence between the fitness landscape and the information on the fitness obtained from this cyclical application of the algorithm's operators, the SB contribution during the search for optima cannot be easily unveiled if not by means of a specific objective function capable of nullifying such interaction over multiple optimisation runs. The $f_{0}$ function, first introduced in [7], serves this purpose and can be used to decouple these effects, thus separating the SB component, arising from algorithmic design choices, from the main driving force represented by the sampled difference in the fitness landscape. $f_{0}$ is a 'truly' random problem, having the simple analytical representation reported below ${ }^{1}$ :

$$
f_{0}:[0,1]^{n} \rightarrow[0,1], \text { where } \forall x, f_{0}(x) \sim \mathcal{U}(0,1) .
$$

\section{A. Existing methodology for measuring $S B$}

As explained in Section II, a heuristic optimisation algorithm that does not exhibit SB should be able to find randomly placed uniformly distributed optima for the special objective function $f_{0}$ with equal difficulty/ease. Therefore, the problem of determining whether a heuristic optimisation algorithm suffers from SB can be reduced to the problem of checking whether the best solutions found by multiple runs of this algorithm to minimise ${ }^{2} f_{0}$ are uniformly distributed. However, such uniformity check is not a trivial task due to the many forms in which SB can manifest itself for different algorithms (see Section II-B) and under different algorithmic configurations. To complicate things further, challenges encountered while testing for SB depend on the experimental setup used to execute optimisers, e.g., where the number of performed runs might be adequate for classic performancebased assessment but not for detecting SB. Moreover, testing for SB also depends on the allowed computational budget allocated to the algorithm and/or employed termination criterion. Indeed, the study in [8] has shown that when SB emerges during the evolutionary process (for several versions of the Differential Evolution algorithm), it only grows stronger during the remaining evaluations. This means that practitioners using different experimental settings might end up looking at biases of different strengths for the same algorithm, which make direct comparisons unfair. These details can make it difficult to generate a practical and robust procedure for the SB detection.

Up until now, methods to check the uniformity of the distribution of best solutions over multiple runs included visual or statistical inspections, briefly discussed in Sections II-A1 and II-A2 respectively.

1) Visual test: Displaying locations of the best solutions collected from multiple runs in the so-called 'parallel coordinates' [9] appears to be the most effective way for visualising SB over a multidimensional problem [7]. This approach is easily reproducible, graphically valid and hence convenient. However, when a large number of images is generated [10], [11], [8], [12], visual inspection can become too laborious. Such approach is also clearly subjective and therefore not reliable for cases where graphical artefacts or unclear patterns cannot be judged by the naked eye.

2) Statistical test: Let us consider a heuristic optimisation algorithm that was run $N$ times to solve the problem of

\footnotetext{
${ }^{1}$ Throughout this paper we refer to one-dimensional uniform sampling within $[a, b]$ as $\mathcal{U}(a, b)$ and to sampling from the Normal distribution with mean $\mu$ and standard deviation $\sigma$ as $\mathcal{N}(\mu, \sigma)$.

${ }^{2}$ or maximise.
} 
minimising $f_{0}$. At the end of each run $i$, the best solution $\mathbf{x}^{(i)}$ found by the algorithm by the end of the run is recorded, where naturally $\mathbf{x}^{(i)} \in[0,1]^{n}$. The random sample $\left\{\mathbf{x}^{(1)}, \mathbf{x}^{(2)}, \cdots, \mathbf{x}^{(N)}\right\}$ represents the set of best solutions retrieved by the $N$ runs of the algorithm. Assume that $\left\{x_{j}^{(1)}, x_{j}^{(2)}, \cdots, x_{j}^{(N)}\right\}, j \in\{1,2, \cdots, n\}$ was drawn from a probability distribution with a continuous cumulative distribution function $\mathcal{F}_{d}$. A goodness-of-fit test can be used to test the null hypothesis $H_{0}: \mathcal{F}_{d} \sim \mathcal{U}(0,1)$. The Kolmogorov-Smirnov test [13] was first employed with a sample of size $N=50$ and significance level $\alpha=0.01$ in [7]. Subsequently, following the 'power analysis' performed in [14] across three common tests, namely KolmogorovSmirnov, Cramér-Von Mises [15] and Anderson-Darling [16] tests, the latter test was chosen and used in combination with the Benjamini-Hochberg [17] correction method for multiple comparisons to achieve higher statistical power. However, it was noted that the original sample size was not adequate for testing all the single-solution algorithms under investigation. Hence, a higher number $N=100$ of runs had to be used for some algorithms in order to achieve a satisfactory level of statistical power. Similar problems were encountered in a further study on SB in a subclass of Estimation of Distribution Algorithms [18], even when using an aggregated measure of SB defined as the sum of the statistically significant (across all dimensions) test statistics of the Anderson-Darling test.

It was concluded that the described statistical approaches can effectively detect most cases of 'strong' SB but are deficient on other scenarios, including clear 'mild' SB that can be dealt with the visual approach. Reasons for these discrepancies between the two methodologies might be a conservative nature of the employed tests combined with the relatively low sample size. Indeed, more accurate SB detection results could be obtained with $N=600$, as used in [8], instead of $N=100$.

Using a large sample size $(N=600)$ indeed seems to catch bias more often, but still gives no guarantee to detect all different kinds of SB, at any significance level [8]. Even larger sample sizes are necessary for smaller levels of significance, higher desired power and smaller sizes of the deviations to be identified [19]. However, given the limited computational resources to run heuristic optimisation algorithms, it is not always possible to obtain (very) large sample sizes. Therefore, tests better able to detect significant deviations from uniformity given limited sample sizes are desirable for detecting SB. We study this in more detail in Section V-B.

Regardless of the above, there is a clear need for a better automated statistical testing procedure to detect SB readily available to the community as a software package.

\section{B. Known deviations from uniformity due to $S B$}

Formulating a good statistical measure of SB has turned out to be difficult (see Section II-A2) due to a wide range of potential deficiencies of distributions of locations of final points - i.e., in what aspect samples from such distributions deviate from uniformity.
The following deviations have been observed for different kinds of iterative heuristic optimisation algorithms [7], [10], [14], [18], [20], [8]

- in terms of proportion of occupied continuous domain:

- sample values do not span the whole domain - the sample is uniform on an interval smaller than $[0,1]$ ('centre-bias');

- values in a sample cover discrete set of values (regularly spaced) within $[0,1]$ ('discretization-bias');

- in terms of locations of points, sample points exhibit:

- local clustering within the domain ('cluster-bias');

- clustering around several clusters within the domain ('cluster-bias');

- clustering in certain parts of each dimension: on the bounds, in one or both sides of each dimension ('bound-bias');

○ one or more large empty gaps consistently identified in all dimensions ('gap-bias');

- in terms of correlations between different dimensions of the sample points: present or not.

\section{BIAS toolbox}

Our proposed BIAS (Bias in Algorithms, Structural) toolbox fills in the gap in the literature in terms of the need for a better automated procedure to detect SB and identify its type. In this paper, we will discuss the different components which constitute the proposed BIAS toolbox. These components include 39 statistical tests and a procedure to aggregate them for detecting SB (Section III), a Random Forest model to identify the type of structural bias (Section VI), the code for the used $f_{0}$ (which was described in Section II) and the code for the generation of the statistical bias decisions and plots (of which an example is illustrated in Figure 3).

The BIAS toolbox is available as a python package at [4]. It provides a clear decision on whether or not structural bias present in the sample of final positions provided by the user and, in case some SB is found, an assessment on the possible type of SB observed in the sample using random forest models. In addition to the structural bias detection, the toolbox also contains the needed functionality to sample data from the scenarios in Table I, thus providing the means to benchmark other statistical test for detecting structural bias.

\section{TESTS}

As explained in Section II-A2, the goodness-of-fit test previously adopted for detecting SB (Anderson-Darling) is deficient on several cases where SB is clearly visible even with a sample size of 100 . More powerful tests are therefore desirable. This section introduces existing and newly proposed goodness-of-fit tests evaluated in this paper for the purpose of detecting SB in samples of limited size. This wide selection of tests includes both classical statistical tests and other tests that have demonstrated competitive power for testing uniformity in the statistical literature [21], [22]. All of these tests are used to test the null hypothesis $H_{0}: \mathcal{F}_{d} \sim \mathcal{U}(0,1)$ introduced in Section II-A2. 


\section{A. Tests per dimension}

The following tests are designed to work on an individual dimension. However, by aggregating all data we can run these test on a sample size which is effectively $n$ times larger. If these tests are run on a per-dimension basis, correction strategies need to be applied to deal with the multiplecomparison problem. For this purpose, the Benjamini-Holberg (BH) method was proposed originally, since it is less stringent than the standard Bonferroni method. However, in this work, we will also investigate the effects of other multiple comparison correction methods, which will be discussed in Section V-D.

1) 1-spacing (1-spacing): [23], [4] To detect cases where the bias takes the form of large spacing differences or clustering in each dimension, we can test the distribution of distances between consecutive points (or points with $m$ neighbours between them, $m \in\{1,2,3\}$ here and in two subsequent tests). Such distribution can then be compared to the distribution from a large sample of truly uniform random samples using a 2-sample $\mathrm{KS}$ test. This and two subsequent tests in the list belong to a wider class of tests called $m$-spacing.

2) 2-spacing (2-spacing): [23], [4] See III-A1.

3) 3-spacing (3-spacing): [23], [4] See III-A1.

4) Sample Range (range): [4] The range taken up by the samples in each dimension can be useful in detecting SB where the points are far removed from the boundaries. The same can be done using the sample extrema (next two items).

5) Sample Minimum (min): [4] See III-A4 for explanation.

6) Sample Maximum (max): [4] See III-A4 for explanation.

7) Anderson-Darling $(A D)$ : [16], [24] is the most commonly used test for checking uniformity, and as such the test proposed to detect SB in [18].

8) Transformed Anderson-Darling ( $t A D)$ : To boost the power of the $A D$ test in some of the most common cases of $\mathrm{SB}$, where the bias occurs near either the boundaries or the centre of the space, a transformation can be applied to the samples by taking their distance to the nearest boundary [20], [24]. This can then be tested using an $A D$ test with domain $\left[0, \frac{1}{2}\right]$.

9) Shapiro (Shapiro): Instead of testing the samples for uniformity directly, we can transform them to their normal counterpart, and check for normality using tests like the Shapiro test [25], [26].

10) Jarque-Bera (JB): This test, obtained through the use of the Lagrange multiplier test on the Pearson family of distributions [27], [28], is also applied to transformed samples to test for their normality.

11) Minimum Linear Distance ( $L D-m i n)$ : [4] The minimum distance between a set of samples and a linearly uniform distribution using the same range and sample size.

12) Maximum Linear Distance ( $L D-$ max): [4] The maximum distance between a set of samples and a linearly uniform distribution using the same range and sample size.

13) Kurtosis (Kurt): This test measures how differently the tails of a distribution are shaped, compared to the tails of the normal distribution. Kurtosis is defined as the fourth standardised central moment, of the random variable of the probability distribution [29], [26]. Note that this test is applied after transforming the data to its normal counterpart.

14) Minimal Minimum Pairwise Distance (MPD-min): [4] The minimum pairwise distances between neighbours. If the minimum distance between neighbours is too small, this can indicate dense clusters.

15) Maxaximal Mininimum Pairwise Distance (MPD-max): [4] Same as $M P D-m i n$ but now comparing the maximum instead. If the maximum distance between neighbours is too large, this indicates uncovered areas of the objective space.

16) Wasserstein distance (Wasserstein): [4] Originally proposed for optimal allocation problems [30] and rigorously formulated by Kantorovitch in [31], this metric is used to measure the distance between two probability distributions defined on a metric space. This result is exploited in this study to build the Wasserstein test for uniformity.

17) Neyman-Smooth test (NS): Neyman [32], [33] constructed his smooth tests specifically to test for the continuous uniform distribution.

18) Kolmogorov-Smirnov (KS): [13], [24] is a nonparametric test based on the maximum distance between the empirical cumulative distribution function (ECDF). While this test is most commonly used to compare 2 samples, it can also be used for goodness-of-fit testing.

19) Cramer-Von Mises (CVM): [34], [22] is similarly based on the expected squared difference between the ECDF and true CDF function of the null distribution.

20) Durbin (Durbin): To test for uniformity, the Durbin $C_{n}$ test [35] compares the cumulated periodogram graph of the residuals from a least-squares regression with the Kolmogorov-Smirnov limits. This is based on previous results showing that when the employed test statistic is calculated with a generic number $2 m+1$ of samples then it is distributed as the mean of $m-1$ independent uniform variables [36], [22].

21) Kuiper (Kuiper): This is an extension of the standard Kuiper test obtained by replacing the original distribution with the Pyke's modified empirical distribution function as proposed in [37] and implemented in [22]. This makes the test suitable for showing that a population has a prescribed continuous distribution function, including uniform.

22) 1st Hegazy-Green (HG1): This test refers to the variant of the Hegazy-Green tests for uniformity proposed in [38] and implemented in [22] employing the $T_{1}$ test statistic defined as the average of the absolute differences between samples and their expected value.

23) 2nd Hegazy-Green (HG2): This is the second variant of the Hegazy-Green test in [38], [22] employing the $T_{2}$ test statistic defined as the average of the squared differences between samples ans their expected value.

24) Greenwood (Greenwood): This test is based on a spacing statistic that can be used to evaluate events in time or locations in space by testing how the intervals between them are distributed [39], [22].

25) Quesenberry-Miller (QM): This test [40], [22] is a modification of the Greenwood test [39] which additionally considers the co-occurrence of extreme squared spacing distances. 
TABLE I

OVERVIEW OF PARAMETERISED DATA SAMPLING SCENARIOS IN $[0,1]$,

THE TOTAL OF 194 / 249 SCENARIO SETTINGS (PER DIMENSION / ACROSS DIMENSIONS), PER CONSIDERED SAMPLE SIZE.

\begin{tabular}{|c|c|c|c|c|c|}
\hline scenario name & how sampled & parameter 1 & parameter 2 & settings & diagnosis $^{3}$ \\
\hline Uniform ${ }^{4}$ & sample full sample size via $\mathcal{U}(0,1)$ & - & - & 1 & no bias \\
\hline Cut Uniform 5 & $\begin{array}{l}\text { subscenario 1: sample full sam- } \\
\text { ple size via } \mathcal{U}\left(z_{c}, 1\right) \text {, subscenario } \\
\text { 2: sample full sample size via } \\
\mathcal{U}\left(\frac{z_{c}}{2}, 1-\frac{z_{c}}{2}\right)\end{array}$ & $\begin{array}{l}\text { fraction cut } z_{c} \in\{0.01 \\
0.025,0.05,0.1,0.2\}\end{array}$ & & $10 / 10$ & centre-bias \\
\hline Cut Normal ${ }^{6}$ & $\begin{array}{l}\text { sample full sample size via } \\
\mathcal{N}(\mu, \sigma), \text { remove all points } \\
\text { outside }[0,1] \text { and repeat until full } \\
\text { sample size }\end{array}$ & $\begin{array}{l}\sigma \in\{0.1,0.2,0.3,0.4 \\
0.5\}\end{array}$ & $\mu \in\{0.5,0.6,0.7\}$ & $15 / 15$ & centre-bias \\
\hline Inverse Cut Normal & $\begin{array}{l}\text { same as above, but transform to } \\
\text { have most mass at bounds }\end{array}$ & same as above & same as above & $15 / 15$ & bound-bias \\
\hline Cut Cauchy & $\begin{array}{l}\text { similar to Cut Normal but for } \\
\text { Cauchy distribution }\end{array}$ & same as above & same as above & $15 / 15$ & centre-bias \\
\hline Inverse Cut Cauchy & $\begin{array}{l}\text { same as above, but transform to } \\
\text { have most mass at bounds }\end{array}$ & same as above & same as above & $15 / 15$ & bound-bias \\
\hline Loose Clusters & $\begin{array}{l}\text { sample } z_{u} \text { portion of sample size } \\
\text { via } \mathcal{U}(0,1) \text {. For each remaining } \\
\text { point, select an existing point } x_{i} \\
\text { and sample } \mathcal{N}\left(x_{i}, \sigma\right)\end{array}$ & $\begin{array}{l}\text { fraction of } \text { uniform } \\
\text { samples } z_{u} \in\{0.1 \\
0.25,0.5\}\end{array}$ & $\begin{array}{l}\sigma \in\{0.01,0.02,0.05 \\
0.1\}\end{array}$ & $12 / 12$ & cluster-bias \\
\hline Gaps & $\begin{array}{l}\text { sample full sample size via } \\
\mathcal{U}(0,1) \text {, select } n_{c} \text { sampled points } \\
x_{i} \text {, remove all sampled points } \\
\text { in }\left[x_{i}-r_{g}, x_{i}+r_{g}\right] \text {, resample } \\
\text { missing points outside gaps via } \\
\mathcal{U}(0,1) \text { until full sample size }\end{array}$ & $\begin{array}{l}\text { number of centres } n_{c} \in \\
\{1,2,3,4,5\}\end{array}$ & $\begin{array}{l}\text { gap radius } r_{g} \in\{0.01 \\
0.02,0.03,0.04,0.05\}\end{array}$ & $25 / 25$ & gap-bias \\
\hline Consistent Gaps ${ }^{10}$ & & same as above & same as above & $0 / 25$ & gap-bias \\
\hline Spikes & $\begin{array}{l}\text { randomly sample integers in } \\
{\left[1, n_{s}\right], \text { rescale as uniformly }} \\
\text { placed spikes in }[0,1]\end{array}$ & $\begin{array}{l}\text { number of spikes } n_{s} \in \\
\{25,50,100,150,200 \\
250,500,1000\}\end{array}$ & - & $8 / 8$ & $\begin{array}{l}\text { discretization- } \\
\text { bias }\end{array}$ \\
\hline \multicolumn{6}{|c|}{${ }^{3}$ See Section II-B } \\
\hline \multicolumn{6}{|c|}{${ }^{4}$ Sanity check, excluded from the tests } \\
\hline \multirow{2}{*}{\multicolumn{6}{|c|}{$\begin{array}{l}5 \text { Two subscenarios: } 1 \text {. modify only min (equivalent to varying only max, so don't do both to save time); } 2 \text {. modify both min and max at the same time, } \\
\text { with the same parameter setting (half cut on both sides) } \\
{ }^{6} \text { Vary } \mu \text { only to one side since it is equivalent to the other side }\end{array}$}} \\
\hline & & & & & \\
\hline \multirow{2}{*}{\multicolumn{6}{|c|}{$\begin{array}{l}{ }^{7} \text { For across-dimension tests, need to differentiate between same cluster-centres in each dimension (Consistent Clusters) vs. different gaps } \\
\text { (Clusters) } \\
{ }^{8} \text { Only used in across-dimension tests, as it is equivalent to Clusters per-dimension }\end{array}$}} \\
\hline & & & & & \\
\hline \multicolumn{6}{|c|}{$\begin{array}{l}{ }^{9} \text { For across-dimension tests, need to differentiate between same gaps in each dimension (Consistent Gaps) vs. different gaps (Gaps) } \\
10 \text { Only in across-dimension tests, as it is equivalent to Gaps per-dimension }\end{array}$} \\
\hline
\end{tabular}

26) Read-Cressie ( $R C)$ : This test [41], [22] belongs to a family of goodness-of-fit tests based on the power divergence statistic.

27) Moran (Moran): This test [42], [22] operates via the distribution of the sum of squares of intervals into which the domain is divided, using a homogeneity of variances test.

28) 1st Cressie (Cressie1): This test [43], [22] is based on the logarithm of $\mathrm{m}$-spacing gaps.

29) 2nd Cressie (Cressie2): This test [44], [22] operates via the family of statistics based on m-spacing gaps, obtained by summing a suitably regular function of each spacing gap.

30) Vasicek (Vasicek): As entropy can be used to characterise distributions, this test uses an estimate of entropy based on higher order $(m>1)$ m-spacings to test for uniformity
[22]. Entropy had been originally adopted by Vasicek [45] for testing the hypothesis of normality.

31) Swartz (Swartz): Instead of using entropy, Swartz [46], [22] uses the Kullback-Leibler information to derive a statistic to test for uniformity. This statistic is also based on $m$-spacings.

32) Morales (Morales): This test [47], [22] compares an empirical and a hypothetical distribution based on the limit laws for a statistic based on $\phi$-disparity [48], [49] of $m$ spacings.

33) Pardo (Pardo): Informational Energy is a measure of certainty, related to the Shannon Entropy, which is a measure of uncertainty. This test uses $m$-spacings to estimate 
Informational Energy as a test statistic for the hypothesis of uniformity [50], [22].

34) Marhuenda (Marhuenda): This test [21], [22] uses a quantile-based divergence statistic based on Cressie and Read's power divergence statistics [51].

35) 1st Zhang (Zhang1): Zhang [52] proposed a general parametrized test statistic that can be used to derive both classical goodness-of-fit test statistics such as Anderson-Darling [16] and Kolmogorov-Smirnov [13] and other new goodnessof-fit test statistics that were demonstrated to be more powerful [52]. Zhangl is derived based on this parametrized test statistic using likelihood ratio statistics [52], [22].

36) 2nd Zhang (Zhang2): This test statistics [52], [22] is derived in a similar way to Zhangl, but is based on an approximation.

\section{B. Tests across dimensions}

In addition to the tests which work on a per-dimension basis, we can also perform tests on the full set of 30-dimensional data at once. This can be done by grouping together the samples or distances and performing the same test as the per-dimension testing on the aggregated data. For this purpose, we use all tests discussed above, with the exception of the sample limitsbased tests, LD, MPD and Wasserstein tests. It can also be done by using across-dimension tests. In this work, we use the following across-dimension tests:

1) The mutual information (MI): The mutual information [53], [54] between variables of a random distribution should be close to zero. If the MI between two variables is higher, this suggests bias towards specific values in the domain shared by these two variables (dimensions). The median mutual information is the median over all dimensions.

The mutual information between two Random Variables $U$ and $V$ is defined as:

$$
M I(U, V)=\sum_{i=1}^{|U|} \sum_{j=1}^{|V|} \frac{\left|U_{i} \cap V_{j}\right|}{N} \log \frac{N\left|U_{i} \cap V_{j}\right|}{\left|U_{i}\right|\left|V_{j}\right|}
$$

2) Maximum Minimum Pair-wise Distance (MMPD): [4] This test is the maximum distance between two neighbouring points in a (multi) dimensional space. This distance should not be significantly different from the same distance metric over a random uniform sampling. A significant higher distance means that there is a region in the search space where the algorithm is more attracted to (and subsequently a region that is avoided). In another words, bias.

3) Maximum Difference per Dimension between a linear uniform distribution (MDDLUD): [4] This test is the multidimensional equivalent of the $L D$-max test, where we aggregate either using the maximum or median across all 30 dimensions.

\section{Critical values for all tests}

To determine rejection based on the test statistic, we need to either calculate the corresponding p-values, or check if the test statistic exceeds the corresponding critical value. Several of the test we include calculate the p-value by default, but for the

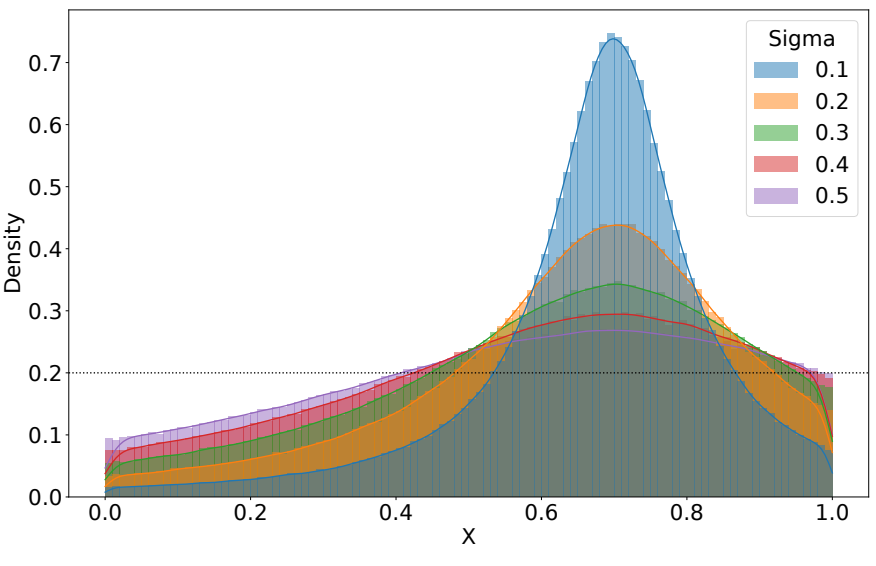

Fig. 1. Empirical Density Distribution for the Cut Cauchy scenario, with varying $\sigma$, and $\mu=0.7$ (based on 1000000 samples each). The dotted line shows the theoretical density of the uniform distribution.

others we will use the critical values. To get accurate estimates of these values, we use a 100000 samples Monte Carlo simulation of the test statistic under the uniform distribution, from which we determine the $\alpha$-quantiles for $\alpha \in\{0.01,0.05\}$. The Monte Carlo test is a well known procedure for implementing hypothesis tests [55]. It enables calculating the critical values when the true (sampling) distribution of the test statistic is unknown. The resulting critical values calculated using this procedure are available at [56].

\section{Methodology}

To effectively judge the performance of the proposed tests for different types of SB, we have defined a large portfolio of scenarios according to which we can generate arbitrary number of samples. This set of scenarios is chosen in such a way that all common types of SB discussed in Section II-B are represented. Additionally, these scenarios are parameterised to control the level of bias, which enables us to better judge the robustness of tests.

\section{A. Portfolio of scenarios}

We choose the scenarios to include based on the most common types of structural bias (see Section II-B) as observed in previous papers:

- bias towards the centre of the search space,

- bias towards the bounds of the search space,

- bias towards certain parts of the search space forming clusters,

- bias towards avoiding certain parts of the search space, creating gaps and

- poor discretization.

In total, this gives us 11 distinct scenarios (13 for the acrossdimension case) - the full list these scenario definitions and their parameters used in this paper is shown in Table I. An example of the density of several parameterizations of scenario Cut Normal is shown in Figure 1.

In Figure 2, we show that there exists minimal overlap between the densities of the different parameterizations of these scenarios by collecting 10000 samples and performing 


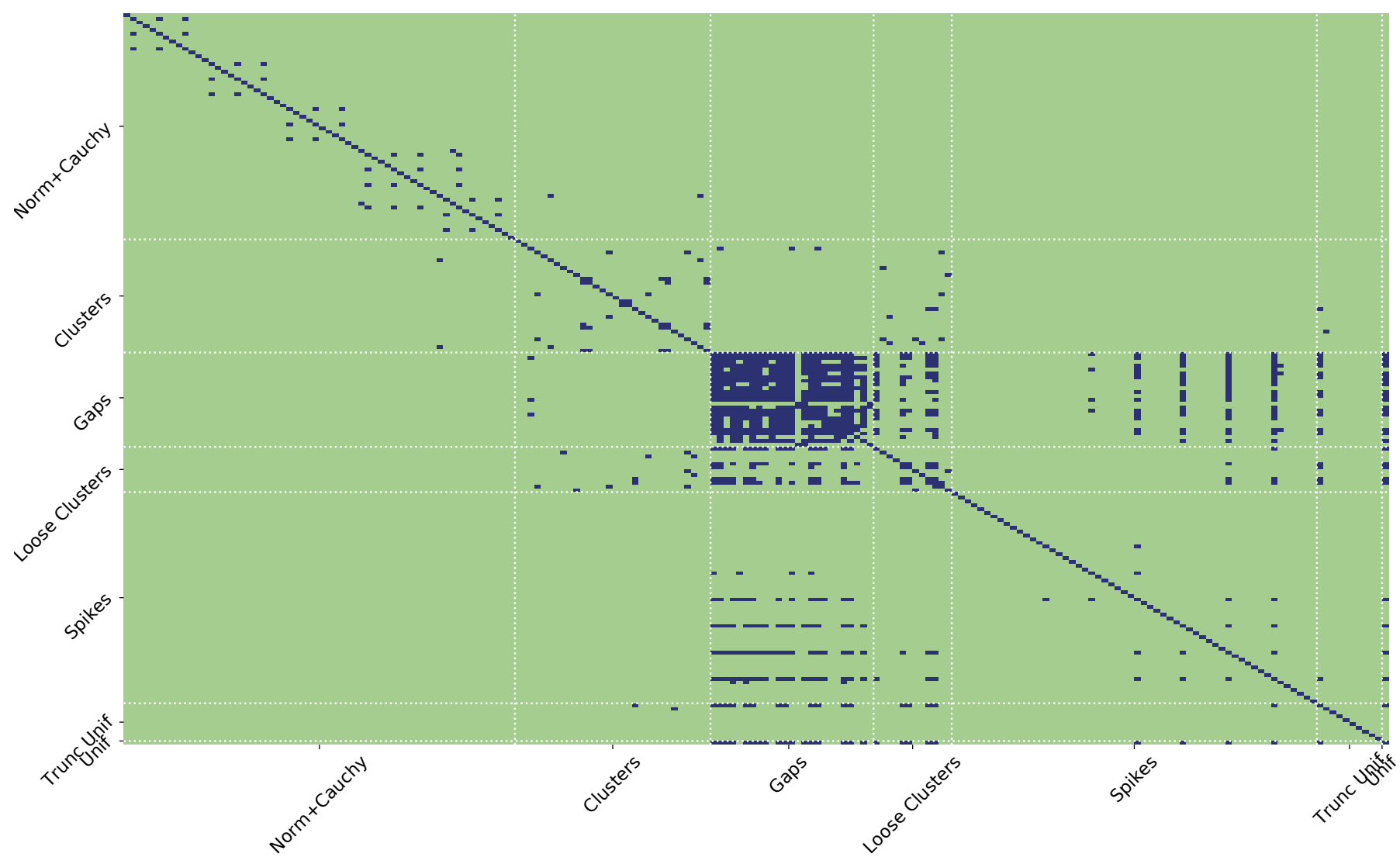

Fig. 2. Confusion matrix via the KS-test between all scenarios. Blue indicating combinations which are not distinguishable based on the 2-sample KS-test for $\alpha=0.01$. Used sample size is 100 , with 1000 sets of samples generated for each scenario, aggregated into a $1 \mathrm{D}$ sample. The white lines divide the types of scenario. Norm+Cauchy group contains all Cut and Inverse scenarios based on Normal and Cauchy distributions.

pairwise $K S$ tests. Note that these samples are aggregations of multiple independent dimensions, so for Gaps this means that each set of 100 samples has its own gap-centres, which explains why it is seen as similar to Uniform.

In total, this gives us 194 scenarios to consider in the perdimension case, and 249 scenarios in the across-dimension case. For each of these scenarios, we generate data with sample sizes $\{30,50,100,600\}$. In the per-dimension case, we collect 1500 independent sets of samples for each use-case, while the across dimension cases all use 100 sets of 30 -dimensional samples.

For each of the generated sets of samples, we apply the corresponding test-battery from Section III with $\alpha \in$ $\{0.05,0.01\}$ : 36 tests for per-dimension case and 32 for the across-dimension case. Using this setup, we thus collect $194 \cdot 1500 \cdot 4 \cdot 36=4.19 \times 10^{7}$ test statistics $/ \mathrm{p}$-values for the per-dimension tests, and $249 \cdot 100 \cdot 4 \cdot 32=3.19 \times 10^{6}$ for the across-dimension tests.

We show an example of the set of statistical tests applied to an instance of the Cut Normal scenario in Figure 3. This figure shows the rejections for each dimension individually, as well as the corresponding sample on which this decision is based. This visualisation is available as part of the toolbox as described in Section II-C, and provides a visual way to inspect the structural bias present in the scenario.

For the analysis on the per-dimension test, we do not directly apply multiple correction methods. However, in section V-D, we will analyse the effect of different correction methods on the overall false positive rate and select which method to use in practice.

\section{AnAlysis OF STATISTICAL TESTS APPLiED TO SCENARIOS}

This section analyses the statistical tests presented in Section III in terms of their suitability to be included in the BIAS toolbox for the purpose of detecting structural bias.

\section{A. Robustness of tests to scenario parameters}

To analyse the effect of different parameterizations of the selected scenarios on the difficulty of detecting bias, we can consider the overall number of rejections for a single test across all parameter settings. An example for the Inverse Cauchy scenario is shown in Figure 4. In this figure, we see clearly that extreme parameter settings (highly shifted mean and low variance) are always detectable by the $A D$ test, while the $t A D$ test performs much better when the distribution is centred.

While this granular view of results can give important insights, it is impractical to use this low-level view to investigate the different impacts of our experiment settings on the final rejections. For the sake of completeness, the figures for all 

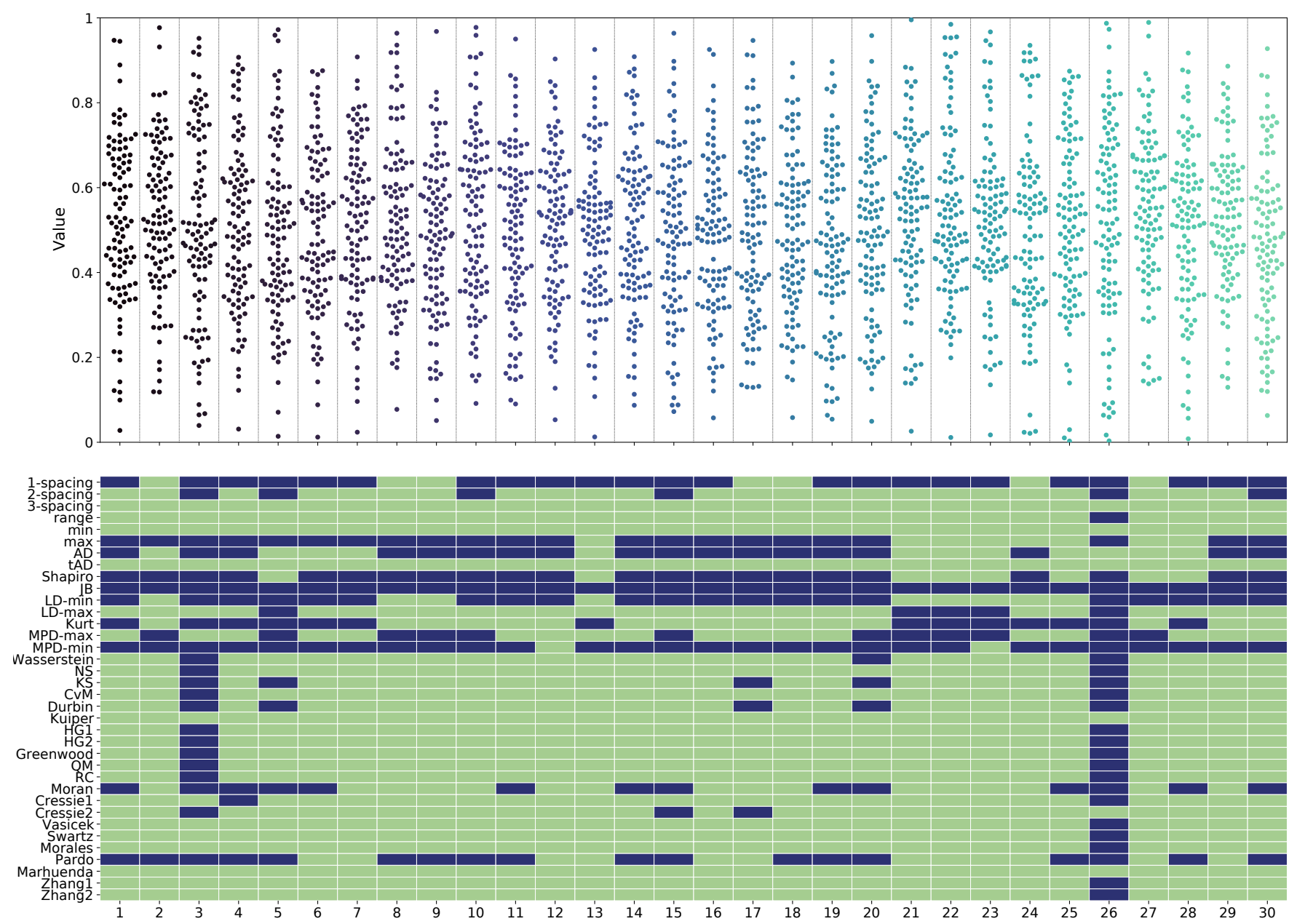

Fig. 3. Example of an instantiation of the Normal Cut scenario with $\mu=0.5$ and $\sigma=0.2$, with 100 samples in each of 30 dimensions. The top figure shows the assumed distribution of the final positions in each dimension. Jitter is applied to vertically overlapping points. The colour scheme is used to highlight different dimensions. The binary heatmap in the bottom shows in green which tests reject the null-hypothesis of uniformity per dimension with $\alpha=0.01$ (no multiple comparison correction applied).
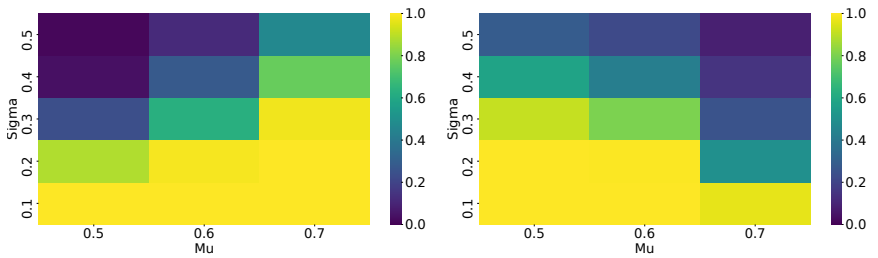

Fig. 4. Fraction of rejected cases for the $A D$ (left) and $t A D$ (right) tests on the Cut Cauchy scenario, based on its parameterizations. The used sample size is 100 , with $\alpha=0.01$ and no multiple comparison correction applied.

scenarios and all experimental settings have been uploaded at [57].

\section{B. Sample size}

To study the impact of the available sample size on the overall performance of different statistical tests, we can aggregate the number of rejections over all parameterizations of each scenario. This allows us to show the fraction of cases of a scenario which are rejected by each test given a certain sample size. Figure 5 shows this for the Cut Normal scenario. From this figure, we can see that the effect of sample size is not the same across all tests. As an example, the $A D$ test has a relatively high number of rejections at 30 samples, but doesn't reach the same precision as other tests when increasing sample size to 600. This indicates that analysis of the performance of the tests should take the number of available samples into account, as this will influence which tests are more distinguishing.

From Figure 5, we can also see that the Moran test significantly outperforms any others on this scenario, but even this test does not reject all cases when the sample size is small. This reinforces the notion that if possible, increasing the sample size is beneficial to the ability to detect less clear cases of structural bias. However, we also note that for most scenarios, a sample size of 50 seems to be sufficient to detect the presence of structural bias. While increasing the sample size would increase the ability to detect less obvious cases of SB, $N=50$ should be able to correctly identify the most blatant ones.

\section{Overall analysis}

With the rejection data, we can investigate the interplay between statistical tests and the scenarios, in order to find 


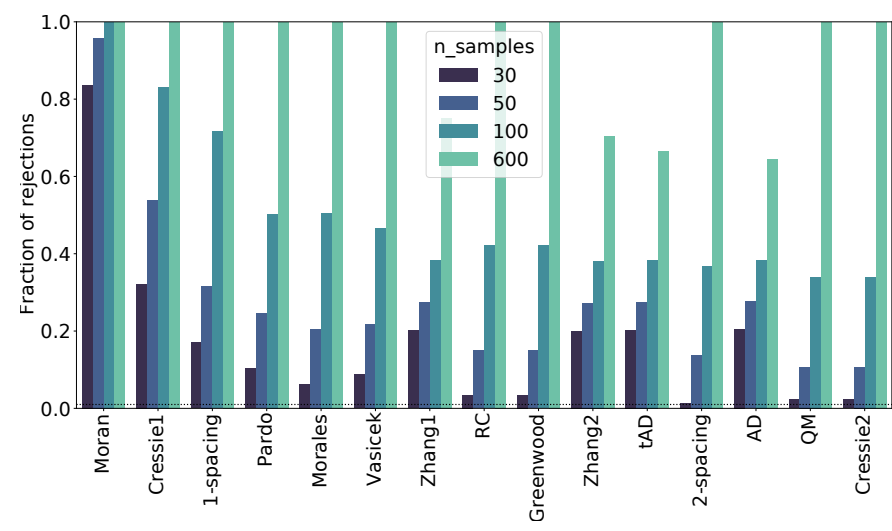

Fig. 5. Fraction of rejections for each test on the Shifted Spikes scenario, with $\alpha=0.01$ and no multiple comparison correction method applied. Data is aggregated over all parameterizations of the scenario, as described in Table I. This figure shows 15 tests with the most rejections (when aggregated over the different sample sizes). Note that the negative space over each bar $(1-x)$ is equivalent to the false negative rate of the test.

what set of tests is more suitable to each kind of structural bias. For this analysis, we make use of the concept of Shapley values [58] to assess the contribution of each test to a portfolio of tests for finding bias in each type of scenario. In particular, we define the marginal contribution of a test $t$ to a portfolio of tests $T^{\prime} \subset T$ on scenario $S$ as follows:

$$
\begin{aligned}
c\left(t, T^{\prime}, S, n, \alpha\right)= & \sum_{s \in S} \sum_{i=0}^{n} \max _{t^{\prime} \in\left(T^{\prime} \cup\{t\}\right)} \mathbb{1}_{t^{\prime}\left(s_{i}\right)<\alpha}- \\
& \sum_{s \in S} \sum_{i=0}^{n} \max _{t^{\prime} \in T^{\prime}} \mathbb{1}_{t^{\prime}\left(s_{i}\right)<\alpha}
\end{aligned}
$$

where $n$ is the number of realizations $s_{i}$ of scenario $s$. The indicator function $\mathbb{1}$ corresponds to the test $t$ rejecting the null hypothesis with significance $\alpha$ on the data from realization $s_{i}$.

Based on this definition of marginal contribution, we can compute approximate Shapley values by sampling random permutations calculating the marginal contribution for each test at each position within this permutation [59], [60]. This can be formulated as follows:

$$
S(t, S, n, \alpha)=\sum_{r} \sum_{i=0}^{m} c\left(t, T^{\prime}, S, n, \alpha\right): T^{\prime} \subset T,\left|T^{\prime}\right|=i
$$

where $r$ is the number of repetitions used, and $m$ is the maximum size of these permutations, which is introduced to ease with computations and because the impact of larger permutations on the total sum is relatively minor - in this paper, we set $m=10$.

Since the used definition of marginal contribution is commutative, we can sum the individual Shapley values for each scenario to get the overall Shapley values across scenarios. These values are then shown in Figure 6, where we can see that most tests have a very comparable contribution to the overall rejections, with relatively few outliers in both positive (e.g. Moran) and negative (e.g. MPD-min) sense. Because of this, we consider all test to have their uses in the portfolio, and refrain from removing any tests from consideration.

Additionally, we can also consider the per-scenario Shapley values to find a relation between the statistical tests and the scenarios which it can most effectively distinguish. This is

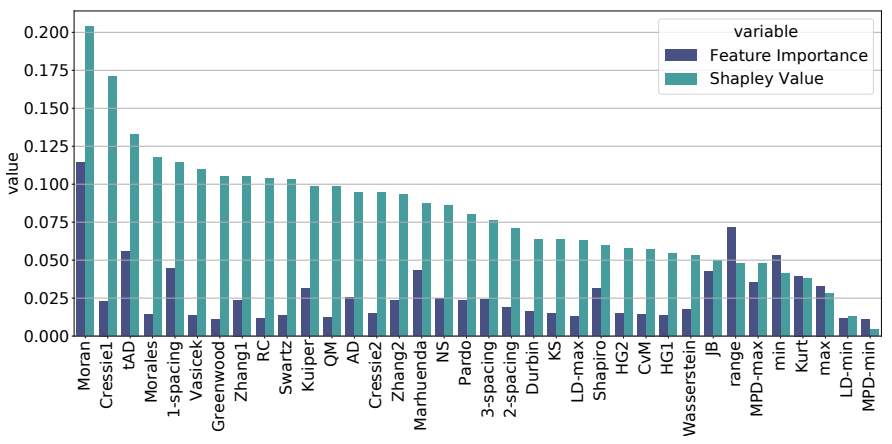

Fig. 6. Approximated Shapley values for all 36 per-dimension tests, based on marginal contribution to the total number of rejections across all scenarios (sample size 100, $\alpha=0.01$ ). Additionally, the feature importance of these tests in the random forest model discussed in Section VI is also shown.

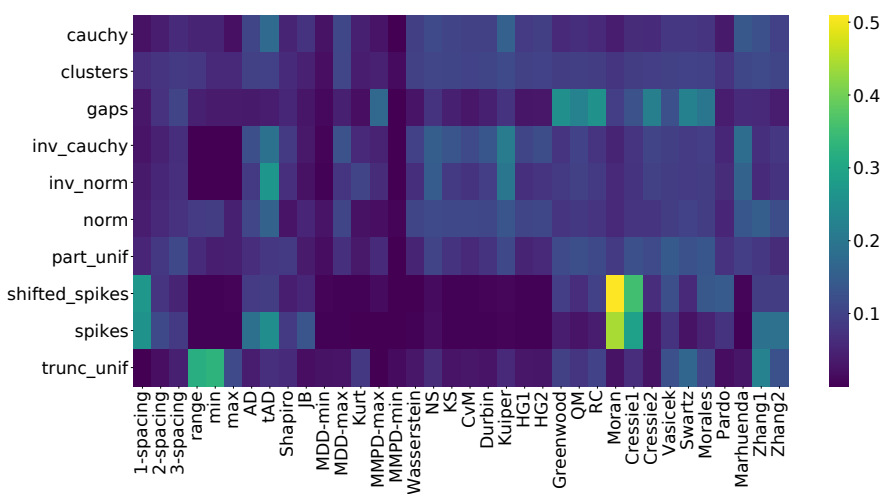

Fig. 7. Approximated Shapley values of all per-dimension tests, based on marginal contribution to the total number of rejections across all parameterization of the used scenarios, with sample size of $100, \alpha=0.01$ and no multiple comparison correction applied. These Shapley values are approximated based on 3600 random permutations.

visualized in Figure 7. There are some clear patterns visible in this figure, namely for the scenarios which mimic poor discretization, where the Moran and Cressiel tests have a very high contribution, while their impact on the other scenarios seems to be relatively minor. This highlights the benefit of having a large portfolio of different statistical test to identify SB.

Overall, we have seen that no single test is clearly preferable over all others. Moreover, an analysis of the Kendall-Tau [61] correlations between the rejections of tests across all scenarios shows that very few tests are highly correlated. Figure 8 shows the correlation heatmaps for two representative settings of sample size and $\alpha$. We can observe relatively higher correlations among some of the tests listed from NS to Greenwood, and among some of the tests listed from $Q M$ to Pardo, in all settings. However, these higher correlations involve very few of the tests, and overall these correlations tend to get slightly weaker as the sample sizes get smaller. Moreover, the correlations among the other tests are very low for the largest sample size of 600 . Therefore, it is likely that different tests are best suited to recognise different types of deviations from uniformity. For this reason, we will include all considered tests in the BIAS toolbox. 

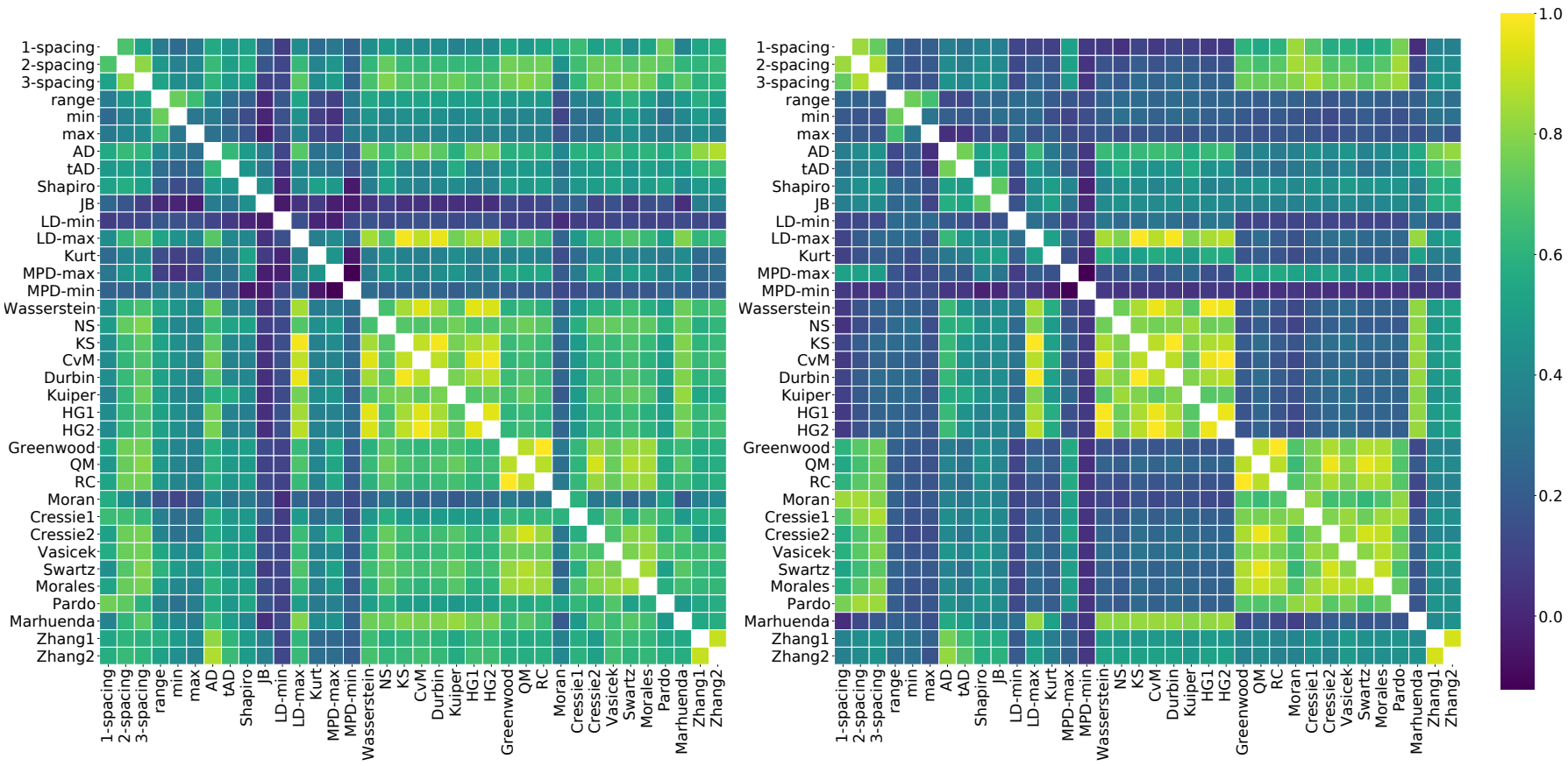

Fig. 8. Cluster plot showing the Kendall-Tau correlations [61] between test rejections on all scenarios, with sample size 50 (left) and 600 (right), $\alpha=0.01$.

\section{Multiple comparison correction methods}

For the per-dimension tests, we should take into account the fact that multiple tests are being done, and thus the p-values should be changed using a correction procedure. For this purpose, we consider 3 methods: BenjaminiHolberg (BH) [62], Benjamini-Yekutieli (BY) [63] and HolmBonferroni (Holm) [64]. For each of these methods, we consider their impact on 30-dimensional samples of the uniform distribution to judge the false positive rate. In Figure 9 on the left, we see the false positive relative the the used $\alpha$ values (defined as at least 1 rejection in the 30D sample). This figure clearly shows the need for a multiple comparison correction method, given that the false positive rate is way over $\alpha$ when using no correction methods. We also note minor differences between the correction methods considered, with BY leading to the lowest false positive rate. Similarly, we can also consider the overall false negative rate as an aggregation over all scenarios, as is done in the right part of Figure 9. This figure shows that the impact of the choice of multiple comparison correction method on the false negatives is relatively minor. Because of this, we set the BY-method as the default, since it is better able to produce false positive rate lower than $\alpha$.

\section{E. Results on correlated samples}

Within the set of scenarios, there are two sets which are enabled only for the across-dimension tests. These scenarios are the consistent version of existing scenarios Gaps and Clusters. In Figure 10, we show the difference in test rejections between these two versions of the scenario. It is clear from the figure that aggregating samples from clusters with different intiantiations on each dimension removes the
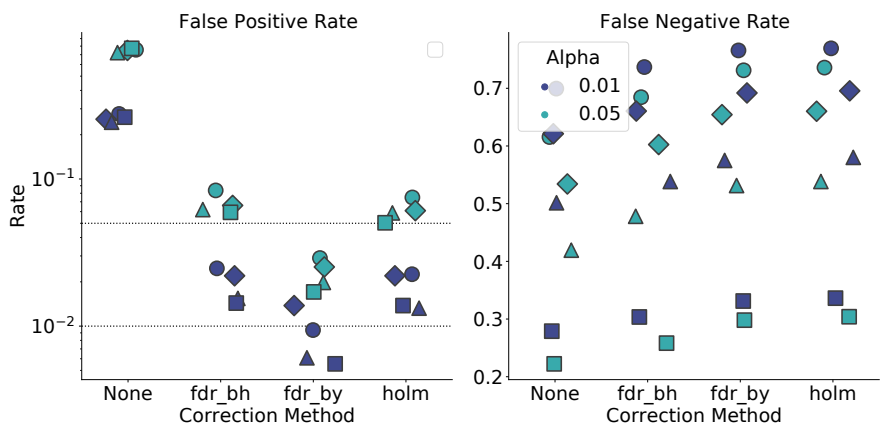

Fig. 9. Evaluation of multiple comparison correction methods: fraction of false positives in 30D samples with different correction methods (on the left) and aggregated fraction of False Negatives across all scenarios of the 6 most distinguishing tests based on Shapley values (on the right). On the left, values above the relevant alpha-thresholds indicate too large false positive rates. On both figures, markers identify the used sample size: $\bigcirc, \diamond, \triangle$ and $\square$ are 30 , 50,100 and 600 respectively. BY method is chosen here.

ability of many tests to detect the bias, while the acrossdimension tests such as MDDLUD do not have this issue. Additionally, since the spacing tests aggregate spacings per dimension instead of samples, their effectiveness is not reduced when the clusters are inconsistent. For the sake of brevity, the other results on the across-dimension tests have are not discussed here, but the relevant figures and data is made available at [56], [57], [65].

\section{Estimation OF THE SB TYPE}

Since we use the results of many statistical tests to find bias in artificially generated samples and different tests may be better at capturing different deviations from uniformity, we can use these tests to not only check if structural bias is present, but also to identify what the most likely form of bias 


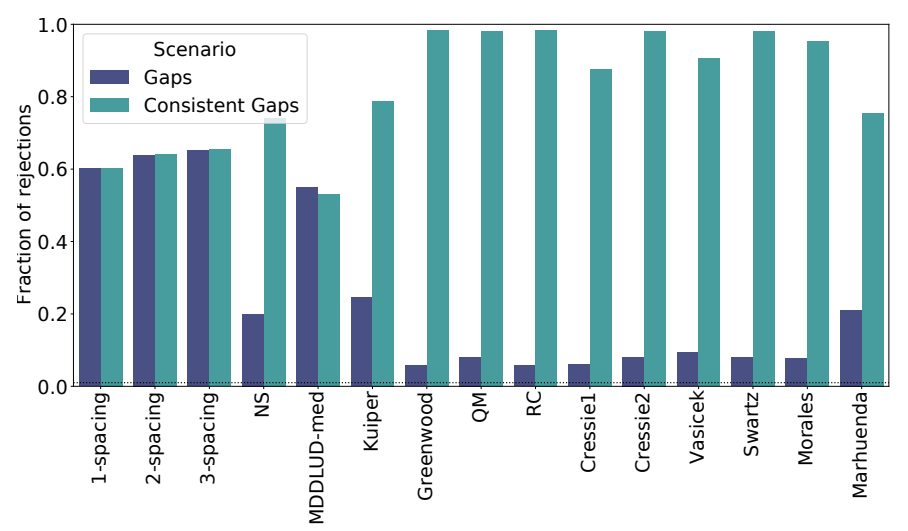

Fig. 10. Overall fraction of rejections across all sample sizes and scenario paramterization of the two versions of the Gaps scenario, using the acrossdimension version of the statistical tests with $\alpha=0.01$ (shown with faint black vertical line). Selection of tests shown is done as the top 15 tests with most total rejections when combining the two bars.

is. This provides an answer to RQ2. To achieve this, we build a random forest (RF) model, which takes as input the testrejections from all per-dimension tests. This is done to allow scaling to arbitrary dimensions while having one model for all sample sizes. Specifically, if we use statistical test values directly, we would need one model per sample size, and a way to aggregate the resulting predictions. Instead, a random forest based on rejections only needs to deal with the aggregation problem.

The data used to train the random forest model consists of the full set of scenario results on all tests, with the output being the scenario-type it comes from. However, if for a specific sample no test rejects the null-hypothesis, these samples are discarded, since we have no evidence of structural bias. This two-stage approach leaves us with 1158000 biased samples, on which we train the RF model with 100 trees and balanced class weights. A confusion matrix created from an 80-20 test split is shown in Figure 11 (F1-score of 0.51). Similarly to Figure 2, we see that the distinction between the Cut Uniform and the other scenarios can be challenging to accurately detect. However, this doesn't have to be an issue for practical detection of $\mathrm{SB}$, since the scenarios misidentified as Cut Uniform might show similar types of bias, even tough their initial creation mechanism is different.

To provide a more practical estimation of SB in our toolbox, we create an additional model to predict the type of bias, as shown in the final column of Table I. These 5 categories are more distinct from each other, removing overlap between some similar classes, i.e. between Spikes and Noisy Spikes. Overall, this model gives us an improved F1-score of 0.70 on a similar 80-20 split.

To use these models in the BIAS toolbox to predict bias of the multi-dimensional test, we need to perform some aggregation across dimensions to transform it into a binary vector. We do this by checking the number of false positive tests in 30D uniform samples. We run 10000 simulations, where we record the maximum number of test rejections by each test. This gives us a total of 92 cases where a test gives 2 rejections, and 2 cases where a test gives 3 rejections. As such,

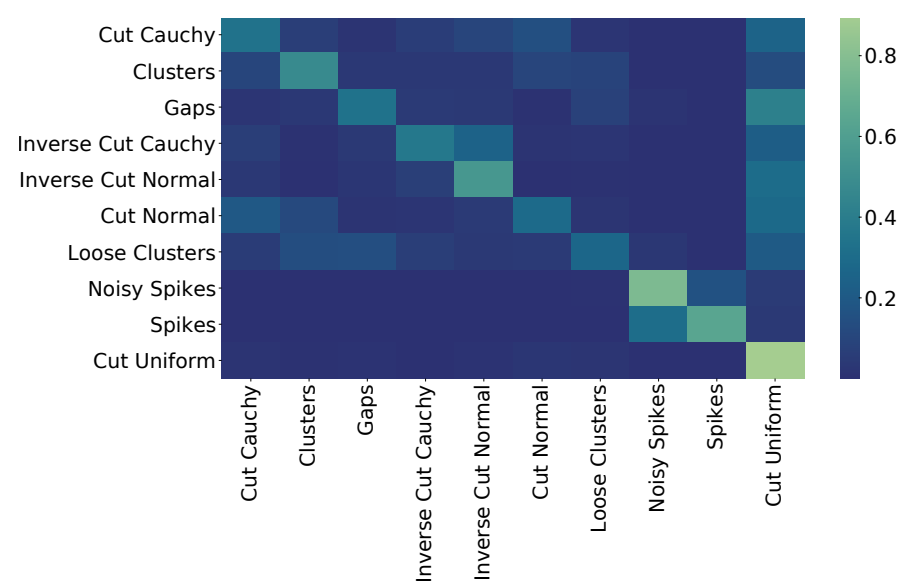

Fig. 11. Confusion matrix for the random forest model trained on test rejections, aggregated over all sample sizes.

we set the threshold for the aggregation of multi-dimensional data to $0.1 \cdot D$. If no test is rejected in this aggregation, we consider the samples to be non-biased.

\section{BENCHMARKING SB OF REAL ALGORITHMIC DATA}

This section benchmarks a range of different heuristic optimisation algorithms by applying the BIAS toolbox, answering RQ3.

\section{A. Data collection setup}

We use data from a heterogeneous pool of heuristics executed over $f_{0}$ at $n=30$ for a maximum of $10000 \cdot n$ fitness functional calls. In total, we consider 409 optimisation heuristics, which fall into the following categories:

- Variants of Differential Evolution (195 configurations, run with $N=100$ )

- Compact optimisation algorithms (81 configurations, run with $N=100$ )

- Single-solution algorithms (60 configurations, run with $N=100)$

- Variants of Genetic Algorithms (96 configurations, run with $N=50$ )

The exact composition of the first three of these categories are described fully in [20]. For the remaining category, the GAs, we make use of a modular structure which gives us a total of 96 configurations obtained by running 8 GA operator combinations with 3 population sizes (i.e. 5, 20 and 100) and 4 strategies for dealing with infeasible solutions (SDIS). Code for the algorithms and the real algorithmic data generation phase can be found in the repository [66] of the employed platform [67]. To create this set of 8 operator combination, we consider combinations of the following options (following the design choices of [7]; further details on the operators available in [68], [69]):

\section{- Selection operators:}

- Roulette wheel selection;

- Tournament selection with tournament size 2

- Crossover operators: 
- Full arithmetic crossover with $\alpha \sim \mathcal{U}(-0.25,1.25)$

- Discrete crossover with crossover rate $C_{r}=0.5$

- Mutation operators:

- Gaussian mutation: $\mathcal{N}(0,0.01)$

- Cauchy mutation: $\mathcal{C}(0,0.01)$

In addition to these operators, we use the following set of SDIS selected amongst those described in [10], [70], [20] ${ }^{11}$ :

- Complete One-sided Truncated Normal strategy (c);

- Dismiss strategy (d);

- Mirror strategy $(\mathrm{m})^{12}$;

- Saturation strategy (s);

- Toroidal strategy (t);

- Uniform strategy $(\mathrm{u})^{12}$.

\section{B. Results}

For each of the considered algorithms, we collect their final positions and feed these into the BIAS toolbox. In Figure 12 (left side), we show the outcome from the RF predicting the type of structural bias present in the different GA configurations (only the biased ones are shown). This shows that there are quite some differences in the detected bias, even within this limited algorithm design space. It is also interesting to note that the population size seems to have a relatively small impact on the type of predicted bias, which seems to be mostly impacted by the operator configuration.

For the single-solution algorithms, we see in the right part of Figure 12 that the strategy of dealing with infeasible solution seems to drastically change the type of detected bias. For example, the Powell algorithm is classified as 'discretization' bias when using mirrored correction, while the classification changes completely with a COTN correction strategy. These differences can give us useful insight into the effect of these SDIS methods on the optimisation behaviour of these algorithms.

\section{CONCLUSION}

Behaviour-based benchmarking is a great way to better understand heuristic algorithms. We have proposed a new behaviour-based benchmark, BIAS, in order to detect different scenarios of structural bias in optimisation algorithms. The BIAS toolbox consists of 36 per-dimension tests and 32 across-dimension tests to detect SB (RQ1). These statistical tests are selected based on elaborate literature research, and some of these tests are specifically designed for this toolbox by the authors. The tests are compared and analysed using different sample sizes and hyper-parameters to detect 11 different scenarios of SB. In addition to the tests, a generator of SB scenarios is provided as well as two machine learning models to predict the scenario of SB using the 11 scenarios mentioned above as well as a more simple grouping of these scenarios since some of these scenarios overlap (RQ2). From the results of the analysis, it is clear that the toolbox performs very well in detecting structural bias in the generated distributions.

\footnotetext{
${ }^{11}$ SDIS methods are applied to newly generated solutions right before their evaluation

${ }^{12}$ not used in GAs, only for algorithms from [20]
}
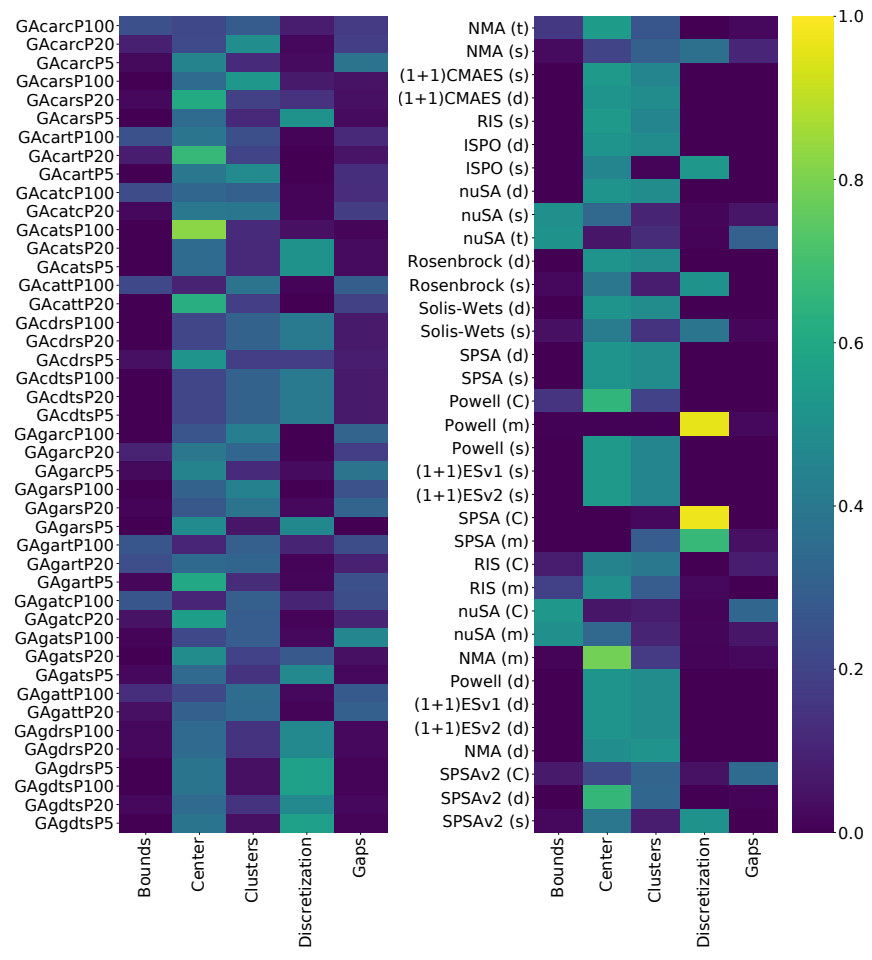

Fig. 12. Predicted SB class probabilities of the biased GA configurations (left, sorted alphabetically) and the biased single-solution algorithms (right), using the random forest model. Names for the GA are structured as mutationcrossover-selection-SDIS-population size. For the single-solution algorithms, the character in brackets refers to the used SDIS.

The machine learning models show some confusion between similar SB scenarios, such as Gaps - Loose clusters, and Spikes - Noisy Spikes, but overall perform well enough to provide suggestions of the type of SB.

In addition we provided the results of BIAS on a large set of optimisation algorithms including variants of Genetic Algorithm and Differential Evolution, compact and single-solution algorithms (RQ3). The results show different kinds of SB in existing heuristic optimisation algorithms. The BIAS toolbox, including the $f_{0}$ function, the statistical tests, the SB scenario generator and the random forest models are provided opensource [4]. We recommend to use the Benjamini-Yekutieli correction method when using BIAS, as this correction method is fast to compute and more conservative than BenjaminiHolberg. We furthermore recommend a minimum sample size of 50 as in most cases it is sufficient to detect SB for all scenarios, as can also be seen from the Genetic Algorithm results. However, some mild SB scenarios could still go undetected. If time and computation power permits, a sample size of 100 would be best.

For future research it would be interesting to explore different machine learning models to see if the predictions of the type of SB can be improved. Additionally, decreasing the number of statistical tests could improve the execution time required to run the benchmark, and may not necessarily decrease accuracy of the BIAS toolbox. 


\section{REFERENCES}

[1] D. Wolpert and W. Macready, "No free lunch theorems for optimization," IEEE Transactions on Evolutionary Computation, vol. 1, pp. 67-82, 1997.

[2] N. Hansen, A. Auger, R. Ros, O. Mersmann, T. Tušar, and D. Brockhoff, "Coco: A platform for comparing continuous optimizers in a black-box setting," Optimization Methods and Software, vol. 36, no. 1, pp. 114 144, 2021.

[3] F. Croce, M. Andriushchenko, V. Sehwag, E. Debenedetti, N. Flammarion, M. Chiang, P. Mittal, and M. Hein, "Robustbench: a standardized adversarial robustness benchmark," arXiv preprint arXiv:2010.09670, 2020.

[4] D. Vermetten, B. v. Stein, F. Carafini, L. L. Minku, and A. V. Kononova, "Bias tooblox," https://github.com/Dvermetten/BIAS.

[5] K. Smith-Miles, D. Baatar, B. Wreford, and R. Lewis, "Towards objective measures of algorithm performance across instance space," Computers \& Operations Research, vol. 45, pp. 12-24, 2014 [Online]. Available: https://www.sciencedirect.com/science/article/pii/ S0305054813003389

[6] O. Mersmann, B. Bischl, H. Trautmann, M. Preuss, C. Weihs, and G. Rudolph, "Exploratory landscape analysis," in Proceedings of the 13th annual conference on Genetic and evolutionary computation, 2011, pp. $829-836$.

[7] A. V. Kononova, D. W. Corne, P. D. Wilde, V. Shneer, and F. Caraffini, "Structural bias in population-based algorithms," Information Sciences, vol. 298, pp. 468-490, 2015.

[8] B. van Stein, F. Caraffini, and A. V. Kononova, "Emergence of structural bias in differential evolution," in Proceedings of the 2021 Genetic and Evolutionary Computation Conference Companion, ser. GECCO '21 Companion. New York, NY, USA: Association for Computing Machinery, July 2021.

[9] A. Inselberg, "The plane with parallel coordinates," The visual computer, vol. 1, no. 2, pp. 69-91, 1985.

[10] F. Caraffini, A. V. Kononova, and D. W. Corne, "Infeasibility and structural bias in differential evolution," Information Sciences, vol. 496, pp. 161-179, 2019.

[11] F. Caraffini and A. V. Kononova, "Structural bias in optimisation algorithms: Extended results," 2021. [Online]. Available: http://dx.doi. org/10.17632/zdh2phb3b4.4

[12] B. van Stein, F. Caraffini, and A. V. Kononova, "Emergence of Structural Bias in Differential Evolution - Source code \& extended graphical results," 2021. [Online]. Available: http://dx.doi.org/10.17632/ pb2bdp2gkp.1

[13] K. Kolmogorov, "Sulla determinazione empirica di una legge di distibuzione, g," 1933.

[14] A. V. Kononova, F. Caraffini, H. Wang, and T. Bäck, "Can single solution optimisation methods be structurally biased?" in 2020 IEEE Congress on Evolutionary Computation (CEC). Glasgow: IEEE, 2020, pp. 1-9.

[15] S. Csorgo and J. J. Faraway, "The Exact and Asymptotic Distributions of Cramer-von Mises Statistics," Journal of the Royal Statistical Society. Series B (Methodological), vol. 58, no. 1, pp. 221-234, 1996.

[16] T. W. Anderson and D. A. Darling, "Asymptotic theory of certain "goodness of fit" criteria based on stochastic processes," The annals of mathematical statistics, pp. 193-212, 1952.

[17] Y. Benjamini, "Discovering the false discovery rate," Journal of the Royal Statistical Society: Series B (Statistical Methodology), vol. 72, no. 4 , pp. 405-416, 2010.

[18] A. V. Kononova, F. Caraffini, H. Wang, and T. Bäck, "Can compact optimisation algorithms be structurally biased?" in Parallel Problem Solving from Nature - PPSN XVI, T. Bäck, M. Preuss, A. Deutz, H. Wang, C. Doerr, M. Emmerich, and H. Trautmann, Eds. Cham: Springer International Publishing, 2020, pp. 229-242.

[19] S. S. Kar and A. Ramalingam, "Is 30 the magic number? issues in sample size estimation," National Journal of Community Medicine, vol. 4, no. 1 , pp. 175-179, 2013.

[20] D. Vermetten, A. V. Kononova, F. Caraffini, H. Wang, and T. Bäck, "Is there anisotropy in structural bias?" in Proceedings of the Genetic and Evolutionary Computation Conference Companion, ser. GECCO '21. New York, NY, USA: Association for Computing Machinery, 2021, p. 1243-1250. [Online]. Available: https://doi.org/10.1145/3449726. 3463218

[21] M. A. Marhuenda, Y. Marhuenda, and D. Morales, "Uniformity tests under quantile categorization," Kybernetes, vol. 34, no. 6, p. 888-901, 2005.
[22] P. L. de Micheaux and V. A. Tran, "Power: A reproducible research tool to ease monte carlo power simulation studies for goodness-of-fit tests in r," Journal of Statistical Software, Articles, vol. 69, no. 3, pp. 1-44, 2016.

[23] R. Pyke, "Spacings," Journal of the Royal Statistical Society: Series B (Methodological), vol. 27, no. 3, pp. 395-436, 1965.

[24] J. Faraway, G. Marsaglia, J. Marsaglia, and A. Baddeley, "goftest: Classical goodness-of-fit tests for univariate distributions." [Online]. Available: https://CRAN.R-project.org/package $=$ goftest

[25] S. S. Shapiro and M. B. Wilk, "An analysis of variance test for normality (complete samples)," Biometrika, vol. 52, no. 3/4, pp. 591-611, 1965.

[26] R Core Team, R: A Language and Environment for Statistical Computing, R Foundation for Statistical Computing, Vienna, Austria, 2013, ISBN 3-900051-07-0. [Online]. Available: http://www.R-project. org/

[27] C. M. Jarque and A. K. Bera, "A test for normality of observations and regression residuals," International Statistical Review/Revue Internationale de Statistique, vol. 55, no. 2, pp. 163-172, 1987.

[28] G. Sucarrat, "Autosearch: General-to-specific (gets) modelling." [Online]. Available: https://cran.r-project.org/package=AutoSEARCH

[29] K. Pearson, "Das Fehlergesetz und Seine Verallgemeinerungen Durch Fechner und Pearson. A Rejoinder," Biometrika, vol. 4, no. 1-2, pp. 169-212, 06 1905. [Online]. Available: https://doi.org/10.1093/biomet/ 4.1-2.169

[30] L. V. Kantorovich, "The mathematical method of production planning and organization," Management Science, vol. 6, no. 4, pp. 363-422, 1939.

[31] L. Kantorovitch, "On the translocation of masses," Management Science, vol. 5, no. 1, pp. 1-4, 1958. [Online]. Available: http: //www.jstor.org/stable/2626967

[32] J. Neyman, "Smooth test for goodness of fit," Scandinavian Actuarial Journal, vol. 1937, no. 3-4, pp. 149-199, 1937.

[33] P. Biecek and T. Ledwina, "ddst: Data driven smooth tests." [Online]. Available: https://cran.r-project.org/package=ddst

[34] H. Cramér, "On the composition of elementary errors: First paper: Mathematical deductions," Scandinavian Actuarial Journal, vol. 1928 no. 1, pp. 13-74, 1928.

[35] J. Durbin, "Tests for serial correlation in regression analysis based on the periodogram of least-squares residuals," Biometrika, vol. 56, no. 1 , pp. 1-15, 031969.

[36] J. Durbin and R. Brown, "Tests of serial independence based on the cumulated periodogram," Bulletin of the International Statistical Institute, vol. 42, pp. 1039-1048, 1967.

[37] H. D. Brunk, "On the Range of the Difference between Hypothetical Distribution Function and Pyke's Modified Empirical Distribution Function," The Annals of Mathematical Statistics, vol. 33, no. 2, pp. 525 532,1962

[38] Y. A. S. Hegazy and J. R. Green, "Some new goodness-of-fit tests using order statistics," Journal of the Royal Statistical Society: Series C (Applied Statistics), vol. 24, no. 3, pp. 299-308, 1975.

[39] M. Greenwood, "The statistical study of infectious diseases," Journal of the Royal Statistical Society, vol. 109, no. 2, pp. 85-110, 1946.

[40] C. Quesenberry and F. M. Jr., "Power studies of some tests for uniformity," Journal of Statistical Computation and Simulation, vol. 5, no. 3 , pp. 169-191, 1977.

[41] N. Cressie and T. R. C. Read, "Multinomial goodness-of-fit tests," Journal of the Royal Statistical Society. Series B (Methodological), vol. 46, no. 3, pp. 440-464, 1984

[42] P. A. P. Moran, "The random division of an interval-part ii," Journal of the Royal Statistical Society. Series B (Methodological), vol. 13, no. 1, pp. $147-150,1951$

[43] N. Cressie, "Power results for tests based on high-order gaps," Biometrika, vol. 65, no. 1, pp. 214-218, 1978.

[44] —_ "An optimal statistic based on higher order gaps," Biometrika, vol. 66, no. 3, pp. 619-627, 121979.

[45] O. Vasicek, "A test for normality based on sample entropy," Journal of the Royal Statistical Society. Series B (Methodological), vol. 38, no. 1, pp. 54-59, 1976.

[46] T. Swartz, "Goodness-of-fit tests using kullback-leibler information," Communications in Statistics: Theory and Methods, vol. 21, p. 711-729, 1992.

[47] D. Morales, L. Pardo, M. Pardo, and I. Vajda, "Limit laws for disparities of spacings," Journal of Nonparametric Statistics, vol. 15, no. 3, pp. 325-342, 2003

[48] B. G. Lindsay, "Efficiency versus robutness: The case for minimum hellinger distance and other methods," Annals of Statistics, vol. 22, pp. 1081-1114, 1994. 
[49] M. L. Menendez, D. Morales, L. Pardo, and I. Vajda, "Two approaches to grouping of data and related disparity statistics," Communications in Statistics - Theory and Methods, vol. 27, p. 609-633, 1998.

[50] M. C. Pardo, "A test for uniformity based on informational energy," Statistical Papers, vol. 44, p. 521-534, 2003.

[51] N. Cressie and T. Read, "Multinomial goodness-of-fit tests," Journal of the Royal Statistic Society, vol. Series B, 46, pp. 440-464, 1984.

[52] J. Zhang, "Powerful goodness-of-fit tests based on the likelihood ratio," Journal of the Royal Statistical Society, vol. Series B, 64, p. 281-294, 2002.

[53] C. E. Shannon, "A mathematical theory of communication," The Bell system technical journal, vol. 27, no. 3, pp. 379-423, 1948.

[54] F. Pedregosa, G. Varoquaux, A. Gramfort, V. Michel, B. Thirion, O. Grisel, M. Blondel, P. Prettenhofer, R. Weiss, V. Dubourg, J. Vanderplas, A. Passos, D. Cournapeau, M. Brucher, M. Perrot, and E. Duchesnay, "Scikit-learn: Machine learning in Python," Journal of Machine Learning Research, vol. 12, pp. 2825-2830, 2011.

[55] E. W. Noreen, Computer-Intensive Methods for Testing Hypotheses: An Introduction. Wiley, 1989.

[56] D. Vermetten, A. V. Kononova, F. Caraffini, B. van Stein, and L. Minku, "Bias: A toolbox for benchmarkingstructural bias in the continuous domain - tests statistics and rejections," Sep 2021. [Online]. Available: https://figshare.com/articles/dataset/_/16546041/0

[57] D. Vermetten, F. Caraffini, A. V. Kononova, B. van Stein, and L. Minku, "Bias: A toolbox for benchmarkingstructural bias in the continuous domain - figures," Sep 2021. [Online]. Available: https://figshare.com/articles/figure/_/16546128/0

[58] L. Shapley, "A value for n-person games," in Contributions to the Theory of Games II, H. Kuhn and A. Tucker, Eds. Princeton University Press, 1953, pp. 307-317.

[59] T. van Campen, H. Hamers, B. Husslage, and R. Lindelauf, "A new approximation method for the shapley value applied to the wtc 9/11 terrorist attack," Social Network Analysis and Mining, vol. 8, no. 1, pp. 1-12, 2018.

[60] J. Castro, D. Gómez, and J. Tejada, "Polynomial calculation of the shapley value based on sampling," Computers \& Operations Research, vol. 36, no. 5, pp. 1726-1730, 2009.

[61] M. G. Kendall, "A new measure of rank correlation,” Biometrika, vol. 30, no. 1/2, pp. 81-93, 1938 .

[62] Y. Benjamini and Y. Hochberg, "Controlling the false discovery rate: a practical and powerful approach to multiple testing," Journal of the Royal statistical society: series B (Methodological), vol. 57, no. 1, pp. 289-300, 1995.

[63] Y. Benjamini and D. Yekutieli, "The Control of the False Discovery Rate in Multiple Testing under Dependency," The Annals of Statistics, vol. 29, no. 4, pp. 1165-1188, 2001

[64] S. Holm, "A simple sequentially rejective multiple test procedure," Scandinavian journal of statistics, pp. 65-70, 1979.

[65] D. Vermetten, A. V. Kononova, F. Caraffini, B. van Stein, and L. Minku, "Bias: A toolbox for benchmarkingstructural bias in the continuous domain - code," Sep 2021. [Online]. Available: https://figshare.com/articles/software/_/16546245/0

[66] F. Caraffini, "Population dynamics SOS (PD-SOS)," Apr. 2021 [Online]. Available: https://doi.org/10.5281/zenodo.4678306

[67] F. Caraffini and G. Iacca, "The sos platform: Designing, tuning and statistically benchmarking optimisation algorithms," Mathematics, vol. 8, no. 5, p. 785, May 2020

[68] D. E. Goldberg and J. H. Holland, "Genetic algorithms and machine learning," Machine Learning, pp. 95-99, 1988.

[69] A. E. Eiben, J. E. Smith et al., Introduction to evolutionary computing. Springer, 2003, vol. 53.

[70] A. V. Kononova, F. Caraffini, and T. Bäck, "Differential evolution outside the box," https://arxiv.org/abs/2004.10489, 2020. 